PROCEEDINGS OF THE

AMERICAN MATHEMATICAL SOCIETY

Volume 130, Number 10, Pages 3067-3074

S 0002-9939(02)06443-2

Article electronically published on March 29, 2002

\title{
STABILIZATION OF EVOLUTION EQUATIONS BY NOISE
}

\author{
ANNA A. KWIECIŃSKA
}

(Communicated by Claudia M. Neuhauser)

\begin{abstract}
We consider a deterministic equation of evolution
$X^{\prime}(t)=A X(t) d t$,

in a separable, real Hilbert space. We prove that if $A$ generates a $C_{0}$-semigroup, then this equation can be stabilized, in terms of Lyapunov exponents, by noise.
\end{abstract}

\section{INTRODUCTION}

In [7] we have constructed the first example of a class of partial differential equations being stabilized, in terms of Lyapunov exponents, by noise. In this paper, we provide a sufficient condition for exponential stabilization of abstract evolution equations, generalizing thus the previous example.

First, in a separable, real Hilbert space $H$, we study an equation of evolution of the form

$$
X^{\prime}(t)=A X(t)
$$

with the initial condition $f$ (see Section 2). We assume that $A$ generates a $C_{0^{-}}$ semigroup. For $f \in \mathcal{D}(A), f \neq 0$, the Lyapunov exponent of the deterministic equation is defined as

$$
\lambda^{\operatorname{det}}(f)=\limsup _{t \rightarrow \infty} \frac{1}{t} \log \left\|x^{\operatorname{det}}(t)\right\|,
$$

where by $x^{\operatorname{det}}(t)$ we have denoted the solution of the deterministic problem. Next, we study the Dirichlet problem for a stochastic perturbation of the above equation, namely

$$
d X=A X d t+\sigma \sum_{k=1}^{N} B_{k} X d \beta_{k},
$$

with the initial condition $f$. We assume that $A$ generates a $C_{0}$-semigroup, the operators $B_{k}$ satisfy the assumptions (A1) (A3) and (A4) and $d \beta_{k}$ denote the Itô differentials. For $f \in \mathcal{D}(A), f \neq 0$, the Lyapunov exponents of the stochastic equation are defined as

$$
\lambda^{\mathrm{st}}(f, \omega)=\limsup _{t \rightarrow \infty} \frac{1}{t} \log \left\|x^{\mathrm{st}}(t, \omega)\right\|,
$$

where by $x^{\text {st }}(t, \omega)$ we have denoted the solution of the stochastic problem.

Received by the editors April 2, 2001 and, in revised form, June 1, 2001.

2000 Mathematics Subject Classification. Primary 35K90, 37L55; Secondary 47D06.

This research was partially supported by KBN grant 2 P03A 01616. 
In Theorem 1 we prove an inequality relating the deterministic and stochastic Lyapunov exponents for a fixed initial condition $f$ and almost all $\omega$. Under our assumptions the stochastic Lyapunov exponents turn out to be smaller, almost surely, than their deterministic counterparts. It means that the deterministic system is made more stable by adding a term with white noise. Moreover, there exists $\sigma_{0}$ such that, for $\sigma \geq \sigma_{0}$ all the stochastic Lyapunov exponents are strictly smaller than zero with probability one. This is especially interesting, because it means that for $\sigma$ big enough the stochastic system is exponentially stable, although the deterministic one may be even exponentially unstable. Theorem 2 provides a sufficient condition for stabilization of deterministic evolution equations by noise (see Corollary 1), which is both fairly general and natural in the semigroup approach. We prove that, if the operator $A$ generates a $C_{0}$-semigroup, then the equation of evolution can be made stable by adding a term with white noise, i.e., all the Lyapunov exponents become smaller than zero with probability one.

Finally, we briefly recall the example from [7] and provide another example to demonstrate that the generalization is worthwhile.

The problem of stabilization of ordinary differential equations by noise, in terms of almost sure Lyapunov exponents, has been thoroughly studied (see for instance [2], 3], 4], 9] and [10]). However, for the time being, to our knowledge, there are very few results concerning stochastic stabilization in the infinite-dimensional case. Apart from our previous paper [7], almost sure exponential stabilization has been studied very recently by Caraballo, Liu and Mao (see [5]) and other types of stabilization have been studied by Leha, Maslowki and Ritter (see [8]).

\section{The ABSTRACT PRoblem}

Let $H$ be a separable, real Hilbert space and let $A$ be a linear operator from $\mathcal{D}(A) \subset H$ into $H$. We consider the following deterministic equation of evolution

$$
X^{\prime}(t)=A X(t)
$$

with the initial condition

$$
X(0)=f \in \mathcal{D}(A) .
$$

Following [11] by a solution to the above Cauchy problem we mean an $H$ valued function $x(t)$ such that $x(t)$ is continuous for $t \geq 0$, continuously differentiable, $x(t) \in \mathcal{D}(A)$ for $t>0$ and (11)-(2) is satisfied. If $A: \mathcal{D}(A) \subset H \rightarrow H$ generates a $C_{0}$-semigroup $S(t)$ and if $f \in \mathcal{D}(A)$, the above Cauchy problem has a unique solution $x(t)=S(t) f$. The solution of the above problem will be denoted by $x^{\operatorname{det}}(t)$. For $f \neq 0, f \in \mathcal{D}(A)$, the Lyapunov exponent of the problem (12)-(2) is defined as

$$
\lambda^{\operatorname{det}}(f)=\limsup _{t \rightarrow \infty} \frac{1}{t} \log \left\|x^{\operatorname{det}}(t)\right\| .
$$

Lemma 1. If the operator $A$ generates a $C_{0}$-semigroup $S(t)$, then for any initial value $f$

$$
\lambda^{\operatorname{det}}(f) \leq \lambda,
$$

where $\lambda=$ const $>0$ and does not depend on $f$.

Proof. It follows from the fact that since $S(t)$ is a $C_{0}$-semigroup, there exist constants $\lambda>0$ and $M \geq 1$ such that $\|S(t)\| \leq M \exp (\lambda t)$; see [11], Theorem 2.2, p.4. 
Let us now consider the stochastic problem

$$
\begin{gathered}
d X=A X d t+\sigma \sum_{k=1}^{N} B_{k} X d \beta_{k}, \\
X(0)=f \in H,
\end{gathered}
$$

where

$$
A: \mathcal{D}(A) \subset H \rightarrow H, \quad B_{k}: \mathcal{D}\left(B_{k}\right) \subset H \rightarrow H, \quad k=1,2, \ldots, N,
$$

$\beta_{1}(\cdot), \ldots, \beta_{N}(\cdot), N<\infty$, are independent, real Wiener processes and $d \beta_{k}, k=$ $1, \ldots, N$, denote the Itô differentials. Let us assume the following:

(1) The operator $A$ generates a semigroup $S(t)$ and the operators $\sigma B_{1}, \ldots, \sigma B_{N}$ generate mutually commuting $C_{0}$-groups $S_{k}(t), k=1, \ldots, N$.

(2) For $k=1, \ldots, N, \mathcal{D}\left(\sigma^{2} B_{k}^{2}\right) \supset \mathcal{D}(A)$ and $\bigcap_{k=1}^{N} \mathcal{D}\left(\left(\left(\sigma B_{k}\right)^{*}\right)^{2}\right)$ is a dense subset of $H$, where $\left(\sigma B_{k}\right)^{*}$ denotes the adjoint of $\sigma B_{k}$.

(3) The operator $C=A-\frac{1}{2} \sigma^{2} \sum_{k=1}^{N} B_{k}^{2}, \mathcal{D}(C)=\mathcal{D}(A)$ is the infinitesimal generator of a $C_{0}$-semigroup $S_{0}(t), t \geq 0$.

(4) There exist $\lambda_{0} \in \mathbf{R}$ and bounded operators $K_{1}, \ldots, K_{N}$ such that

$$
\left(\lambda_{0}-C\right) \sigma B_{k}\left(\lambda_{0}-C\right)^{-1}=\sigma B_{k}+K_{k}, \quad k=1, \ldots, N .
$$

Then, if $f \in \mathcal{D}(C)$, the above problem has a unique strong solution, as defined in 6], p. 152 , on any interval $[0, T]$, where $T>0$; see [6], Proposition 6.29 , p. 176, and Theorem 6.30, p. 177.

We will study next an evolution equation of the above form, and we assume that:

(A1) The operators $B_{k}$ are diagonalizable in the same basis and are bounded from above and below, i.e., there exist $\left\{e_{j}\right\}$ a basis of the Hilbert space $\mathrm{H}$ and constants $d_{i}^{k}$, where $k=1, \ldots, N$ and $i=1,2, \ldots$, such that

$$
\left\langle B_{k} e_{i}, e_{j}\right\rangle=d_{k}^{i} \delta_{i j}, \quad k=1, \ldots, N ; \quad i, j=1,2, \ldots,
$$

where $\langle\cdot, \cdot\rangle$ denotes the scalar product in $H$. The assumption that the operators $B_{k}$ are bounded from above and below is then equivalent to the condition that there exist constants $m_{k}>0$ and $M_{k}>0, k=1, \ldots, N$, such that

$$
m_{k} \leq\left|d_{i}^{k}\right| \leq M_{k}, \quad k=1, \ldots, N ; \quad i=1,2, \ldots .
$$

Obviously the operators $B_{k}$ also mutually commute.

(A2) The operator $A$ generates a $C_{0}$-semigroup.

(A3) There exist $\lambda_{0} \in \mathbf{R}$ and bounded operators $K_{1}, \ldots, K_{N}$ such that

$$
\left(\lambda_{0}-C\right) \sigma B_{k}\left(\lambda_{0}-C\right)^{-1}=\sigma B_{k}+K_{k}, \quad k=1, \ldots, N,
$$

where $C=A-\frac{1}{2} \sigma^{2} \sum_{k=1}^{N} B_{k}^{2}$.

(A4) The operators $A$ and $B_{k}, k=1, \ldots, N$, commute.

Proposition 1. The general assumptions (1)-(4) follow from (A1)-(A4).

To prove the above proposition we will need two lemmas.

Lemma 2. The $C_{0}$-semigroups generated by $A$ and $B_{k}$ mutually commute.

Lemma 3. Let $S(t)$ and $T(t)$ be two commuting $C_{0}$-semigroups. We put $R(t)=$ $S(t) T(t)$. Then $R(t)$ is also a $C_{0}$-semigroup.

We omit the proofs, as they are rather straightforward. 
Proof of the Proposition. (1) Follows from Lemma 2 and (A1).

(2) We have $\mathcal{D}\left(B_{k}\right)=\mathcal{D}\left(B_{k}^{*}\right)=H, k=1, \ldots, N$, which implies (2).

(3) Obviously $\mathcal{D}(C)=\mathcal{D}(A)$, where $C=A-\frac{1}{2} \sigma^{2} \sum_{k=1}^{N} B_{k}^{2}$. The semigroup generated by the operator $C$ is given by the formula

$$
S_{0}(t)=S(t) \prod_{k=1}^{N} \exp \left(-\frac{1}{2} t \sigma^{2} B_{k}^{2}\right) .
$$

We have assumed that the operator $A$ generates a $C_{0}$-semigroup. On the other hand, the operators $B_{k}^{2}$ are bounded and defined on the whole space $H$ and therefore $\exp \left(-\frac{1}{2} t \sigma^{2} B_{k}^{2}\right), k=1, \ldots, N$, define $C_{0}$-semigroups. Due to Lemma 2 these semigroups are mutually commuting, so Lemma 3 enables us to conclude that the operator $C$ generates a $C_{0}$-semigroup.

(4) It is the assumption (A3).

So Proposition 6.29 and Theorem 6.30 from [6] can be applied and if $f \in \mathcal{D}(C)=$ $\mathcal{D}(A)$, there exists a unique solution to the problem (3)-(4). This solution is given by the formula

$$
x^{\mathrm{st}}(t)=S_{0}(t) \prod_{k=1}^{N} \exp \left[\sigma B_{k} \beta_{k}(t)\right] f .
$$

For $f \neq 0, f \in \mathcal{D}(A)$, the Lyapunov exponent of the system (3)-(4) is defined as

$$
\lambda^{\mathrm{st}}(f, \omega)=\limsup _{t \rightarrow \infty} \frac{1}{t} \log \left\|x^{\mathrm{st}}(t, \omega)\right\| .
$$

From Lemma 1 and the next theorem it will follow that $\lambda^{\text {st }}(f, \omega)<\infty$ a.s.

Theorem 1. Let $f \in \mathcal{D}(A), f \neq 0$. Under the assumptions (A1)-(A4) the following inequality holds:

$$
\lambda^{\mathrm{st}}(f, \omega) \leq \lambda^{\operatorname{det}}(f)-\sigma^{2} \alpha \quad \text { a.s. }
$$

where

$$
\alpha=-\limsup _{t \rightarrow \infty} \frac{1}{t} \log \left\|\prod_{k=1}^{N} \exp \left[\left(-\frac{1}{2} B_{k}^{2}\right) t\right]\right\|>0 .
$$

It follows that there exists $\sigma_{0}$ such that for $\sigma \geq \sigma_{0}$

$$
\lambda^{\mathrm{st}}(f, \omega)<0 \quad \text { a.s. }
$$

and $\sigma_{0}$ does not depend on $f$.

Proof. We have

$$
\begin{gathered}
\lambda^{\text {st }}(f, \omega)=\limsup _{t \rightarrow \infty} \frac{1}{t} \log \left\|S_{0}(t) \prod_{k=1}^{N} \exp \left[\sigma B_{k} \beta_{k}(t)\right] f\right\| \\
\leq \limsup _{t \rightarrow \infty} \frac{1}{t} \log \left\|\prod_{k=1}^{N} \exp \left[\left(-\frac{1}{2} \sigma^{2} B_{k}^{2}\right) t\right] \prod_{k=1}^{N} \exp \left[\sigma B_{k} \beta_{k}(t)\right]\right\|\|S(t) f\| \\
\leq \limsup _{t \rightarrow \infty} \frac{1}{t} \log \left\|\prod_{k=1}^{N} \exp \left[\left(-\frac{1}{2} \sigma^{2} B_{k}^{2}\right) t\right] \prod_{k=1}^{N} \exp \left[\sigma B_{k} \beta_{k}(t)\right]\right\|+\limsup _{t \rightarrow \infty} \frac{1}{t} \log \|S(t) f\|
\end{gathered}
$$




$$
\begin{gathered}
\leq \lambda^{\operatorname{det}}(f)+\limsup _{t \rightarrow \infty} \frac{1}{t} \log \left\|\prod_{k=1}^{N} \exp \left[\left(-\frac{1}{2} \sigma^{2} B_{k}^{2}\right) t\right]\right\|\left\|\prod_{k=1}^{N} \exp \left[\sigma B_{k} \beta_{k}(t)\right]\right\| \\
\leq \lambda^{\operatorname{det}}(f)+\limsup _{t \rightarrow \infty} \frac{1}{t} \log \left\|\prod_{k=1}^{N} \exp \left[\left(-\frac{1}{2} \sigma^{2} B_{k}^{2}\right) t\right]\right\|+\limsup _{t \rightarrow \infty} \frac{1}{t} \log \left\|\prod_{k=1}^{N} \exp \left[\sigma B_{k} \beta_{k}(t)\right]\right\| .
\end{gathered}
$$

Since the operators $B_{k}, k=1, \ldots, N$, are bounded from above by constants $M_{k}>0, k=1, \ldots, N$, we have

$$
\left\|\prod_{k=1}^{N} \exp \left[\sigma B_{k} \beta_{k}(t)\right]\right\| \leq \prod_{k=1}^{N} \exp \left[\sigma M_{k}\left|\beta_{k}(t)\right|\right],
$$

and hence

$$
\limsup _{t \rightarrow \infty} \frac{1}{t} \log \left\|\prod_{k=1}^{N} \exp \left[\sigma B_{k} \beta_{k}(t)\right]\right\| \leq 0,
$$

since by the strong law of large numbers $\lim _{t \rightarrow \infty} \beta_{k}(t) / t=0$ with probability 1 , $k=1, \ldots, N$ (see [1], page 46).

On the other hand, since all the operators $B_{k}$ are diagonal and there exist constants $m_{k}>0$ and $M_{k}>0$ such that

$$
m_{k} \leq\left\|B_{k}\right\| \leq M_{k}
$$

an easy computation gives the following bounds:

$$
\begin{aligned}
& -\frac{1}{2} \sigma^{2}\left(\sum_{k=1}^{N} M_{k}^{2}\right) \leq \frac{1}{t} \log \left\|\prod_{k=1}^{N} \exp \left[-\frac{1}{2} \sigma^{2}\left(B_{k}^{2}\right) t\right]\right\| \\
= & \sigma^{2} \frac{1}{t} \log \left\|\prod_{k=1}^{N} \exp \left[-\frac{1}{2}\left(B_{k}^{2}\right) t\right]\right\| \leq-\frac{1}{2} \sigma^{2}\left(\sum_{k=1}^{N} m_{k}^{2}\right)
\end{aligned}
$$

and hence there exists $\alpha>0$ such that

$$
\limsup _{t \rightarrow \infty} \frac{1}{t} \log \left\|\exp \left[\left(-\frac{1}{2} \sigma^{2} \sum_{k=1}^{N} B_{k}^{2}\right) t\right]\right\|=-\sigma^{2} \alpha .
$$

Now the first part of the statement of the theorem follows.

The second part follows directly from the first part and Lemma 1

Let us now consider the problem (3)-(4) with $B=\mathrm{Id}$, i.e.,

$$
\begin{gathered}
d X(t)=A X(t) d t+\sigma X(t) d \beta(t), \\
X(0)=f \in \mathcal{D}(A) .
\end{gathered}
$$

We denote by $\lambda^{\text {st }}(f, \omega)$ the Lyapunov exponent of the stochastic problem (8) $-(9)$ and by $\lambda^{\operatorname{det}}(f)$ the Lyapunov exponent of the deterministic problem (1)-(2).

Theorem 2. Let $f \neq 0, f \in \mathcal{D}(A)$. If the operator $A$ generates a $C_{0}$-semigroup, solutions to (11)-(2) and (8)-(9) exist, are unique and we have the following estimate:

$$
\lambda^{\mathrm{st}}(f, \omega) \leq \lambda^{\operatorname{det}}(f)-\frac{1}{2} \sigma^{2} \quad \text { a.s. }
$$


Proof. The existence and uniqueness of solution to the deterministic problem (1)(22) follow from the fact that $A$ generates a $C_{0}$-semigroup. For the stochastic problem it is enough to check that the conditions (A1)-(A4) hold.

The assumptions (A1), (A2) and (A4) are obviously satisfied. We have to verify that (A3) holds. We compute

$$
\left(\lambda_{0} \mathrm{Id}-C\right)=\left(\lambda_{0} \mathrm{Id}-\left(A-\frac{1}{2} \sigma^{2} \mathrm{Id}\right)\right)=\left(\left(\lambda_{0}+\frac{1}{2} \sigma^{2}\right) \mathrm{Id}-A\right) .
$$

Since $A$ generates a $C_{0}$-semigroup the operator $A$ is closed and there exists $\delta \geq 0$ such that the resolvent set $\varrho(A)$ of $A$ contains the ray $(\delta, \infty)$; see 11, Theorem 5.3, p.20. It follows that if $\gamma>\delta$, then $R(\gamma: A)$ is well defined. Therefore, if we take $\lambda_{0}$ satisfying

$$
\lambda_{0}+\frac{1}{2} \sigma^{2}>\delta
$$

we have that

$$
\left(\lambda_{0} \operatorname{Id}-C\right) \sigma \operatorname{Id}\left(\lambda_{0} \operatorname{Id}-C\right)^{-1}=\sigma\left(\lambda_{0} \operatorname{Id}-C\right)\left(\lambda_{0} \operatorname{Id}-C\right)^{-1}=\sigma \operatorname{Id} .
$$

It means that (A3) holds with $K=0$.

We can now apply Theorem 1 . It is easy to check that $\alpha=\frac{1}{2}$.

Corollary 1. If the operator $A$ generates a $C_{0}$-semigroup, the deterministic system (11)-(2) can be stabilized by noise.

Proof. Let us fix $f \neq 0, f \in \mathcal{D}(A)$. Lemma 1 ensures the existence of a constant $\lambda>0$ such that

$$
\lambda^{\operatorname{det}}(f) \leq \lambda
$$

Let $\sigma_{0}$ be such that

$$
\frac{1}{2} \sigma_{0}^{2}>\lambda
$$

then, for $\sigma \geq \sigma_{0}$ we have $\lambda^{\text {st }}(f, \omega)<0$ for almost all $\omega$.

\section{EXAMPLES}

Example 1. Here is the example from 7]. Let us take $H=L^{2}(\mathcal{O})$, where $\mathcal{O}$ is a bounded domain in $\mathbf{R}^{d}$ with a smooth boundary, $A=\Delta+\alpha I d$, where $\alpha$ is an arbitrary constant, $\mathcal{D}(A)=H_{0}^{1}(\mathcal{O}) \cap H^{2}(\mathcal{O})$ and $B=$ Id. It follows from Theorem 2 that

$$
\lambda^{\mathrm{st}}(f, \omega) \leq \lambda^{\operatorname{det}}(f)-\frac{1}{2} \sigma^{2} \quad \text { a.s. }
$$

In fact, in [7, we have obtained a slightly stronger result. We have proved that

$$
\lambda^{\mathrm{st}}(f, \omega)=\lambda^{\operatorname{det}}(f)-\frac{1}{2} \sigma^{2} \quad \text { a.s. }
$$

It follows from the above equality that the stochastic Lyapunov exponents are nonrandom, i.e., that they depend only on $f$ with probability one. We have also demonstrated that the Lyapunov exponents, both deterministic and stochastic, exist as limits.

Constants $\alpha$ and $\sigma$ may be chosen such that the top Lyapunov exponent of the deterministic system is greater than zero, while the top Lyapunov exponent of the stochastic system is smaller than zero. For details see [7]. 
Example 2. Again let $\mathcal{O}$ be a bounded domain in $\mathbf{R}^{d}$ with a smooth boundary and let $H=L^{2}(\mathcal{O})$.

We consider the differential operator of order $2 m$,

$$
A(x, D)=\sum_{|\alpha| \leq 2 m} a_{\alpha}(x) D^{\alpha},
$$

where $a_{\alpha}(x)$ are sufficiently smooth real-valued functions of $x$ in $\overline{\mathcal{O}}$ and

$$
D^{\alpha}=D_{1}^{\alpha_{1}} D_{2}^{\alpha_{2}} \ldots D_{n}^{\alpha_{n}}=\frac{\partial^{\alpha_{1}}}{\partial x_{1}^{\alpha_{1}}} \frac{\partial^{\alpha_{2}}}{\partial x_{2}^{\alpha_{2}}} \ldots \frac{\partial^{\alpha_{n}}}{\partial x_{n}^{\alpha_{n}}} .
$$

We assume that this operator is strongly elliptic, i.e., there exists a $c>0$ such that

$$
(-1)^{m} \sum_{|\alpha|=2 m} a_{\alpha}(x) \xi^{\alpha} \geq c|\xi|^{2 m}
$$

for all $x \in \overline{\mathcal{O}}$ and $\xi \in \mathbf{R}^{d}$.

Let $A(x, D)$ be strongly elliptic in $\mathcal{O}$ and set $\mathcal{D}(A)=H^{2 m}(\mathcal{O}) \cap H_{0}^{m}(\mathcal{O})$. For every $u \in \mathcal{D}(A)$ we define

$$
A u=A(x, D) u \text {. }
$$

Then $-A$ is the infinitesimal generator of a $C_{0}$-semigroup of operators on $L^{2}(\mathcal{O})$. This fact follows from [11, Chapter 7.2.

We can now deduce from Theorem 2 that the deterministic problem

$$
\begin{gathered}
\frac{\partial u}{\partial t}=-A u, \\
u(0)=f \in H^{2 m}(\mathcal{O}) \cap H_{0}^{m}(\mathcal{O}),
\end{gathered}
$$

can be stabilized by noise, i.e., for $B=\mathrm{Id}$ we obtain the estimate

$$
\lambda^{s t}(f, \omega) \leq \lambda^{d e t}(f)-\frac{1}{2} \sigma^{2} \quad \text { a.s. },
$$

which means that for $\sigma$ big enough the stochastic system becomes stable regardless whether the corresponding deterministic system was stable or unstable.

\section{ACKNOWLEDGEMENTS}

The author wishes to thank Professor M. Capiński and Professor S. Peszat for helpful discussions. The author is also indebted to the referee for his valuable comments.

\section{REFERENCES}

1. L. Arnold: Stochastic Differential Equations: Theory and Applications. Wiley-Interscience, Wiley, New York, 1974. MR 56:1456

2. L. Arnold: A new example of an unstable system being stabilized by random parameter noise. Inform. Comm. Math. Chem., 1979, 133-140. MR 81g:60067

3. L. Arnold, H. Crauel and V. Wihstutz: Stabilization of linear systems by noise. SIAM J. Control Optim. 21, 1983, 451-461. MR 84g:93080

4. L. Arnold and P. Kloeden: Lyapunov exponents and rotation number of two-dimensional systems with telegraphic noise. SIAM J. Appl. Math. 49, 1989, 1242-1274. MR 90f:93062

5. T. Caraballo, K. Liu and X. Mao: On stabilization of partial differential equations by noise, Nagoya Math. J. 161, 2001, 155-170.

6. G. Da Prato and J. Zabczyk: Stochastic Equations in Infinite Dimensions. Encyclopedia of Mathematics and its Applications vol.44, Cambridge University Press, Cambridge, 1992.

7. A. A. Kwiecińska: Stabilization of partial differential equations by noise. Stochastic Process. Appl. 79, 1999, 179-184. MR 2000b:35284 
8. G. Leha, B. Maslowski and G. Ritter: Stability of solutions to semilinear stochastic evolution equations. Stochastic Anal. Appl. 17(6), 1999, 1009-1051. MR 2001a:60074

9. E. Pardoux and V. Wihstutz: Lyapunov exponents and rotation number of two-dimensional stochastic systems with small diffusion. SIAM J. Appl. Math. 48, 1998, 442-457. MR 89e:60116

10. E. Pardoux and V. Wihstutz: Lyapunov exponents of linear stochastic systems with large diffusion term. Stochastic Process. Appl. 40, 1992, 289-308. MR 93e:60114]

11. A. Pazy: Semigroups of linear operators and applications to partial differential equations. Applied Mathematical Sciences vol. 44, Springer-Verlag, New York, 1983. MR 85g:47061

Institute of Mathematics, Polish Academy of Sciences, ul. Śniadeckich 8, 00-950 Warszawa, Poland

E-mail address: akwiecin@impan.gov.pl 